\title{
Rapid Manufacturing of Individualized Prosthetic Sockets
}

\author{
Filip Górski ${ }^{\text {* }}$ Ewa Suszek , Radosław Wichniarek', Wiesław Kuczko', \\ Magdalena Żukowska' \\ 1 Poznan University of Technology, Chair of Production Engineering and Management, Poznan, Poland \\ * Corresponding author's e-mail: filip.gorski@put.poznan.pl
}

\begin{abstract}
The paper presents the experimental process of manufacturing individualized prosthetic sockets out of thermoplastic material, using an additive manufacturing technology of Fused Deposition Modelling. The patient was an adult male with an amputated forearm. His stump was 3D scanned using a low-cost 3D scanner in a semi-automated manner. Then, the anthropometric data was used for the creation of a model of a prosthetic socket, which was subsequently manufactured. Three different 3D printers were used, with three different materials (ABS, nylon and PC) and three different sets of process parameters. The paper contains the descriptions of the process, its results and opinions by the patient, leading to the selection of an optimal process course variant.
\end{abstract}

Keywords: prosthesis, fused deposition modelling, 3D printing, additive manufacturing, customization

\section{INTRODUCTION}

The lack of one or both upper limbs is a significant limitation to the human movement performance. This problem concerns a large group of people and can be a result of both birth defects and amputations. The birth limb defects (so-called birth amputations) are results of disturbances occurring in the fetal life, such as stopping of growth or damage of fetus. As regards the amputations, the most frequent causes are [1]:

- vascular diseases and complications - approx. $65 \%$,

- diabetes and its complications - approx. $20 \%$, - injuries - approx. $11.5 \%$.

Despite the great progress of medical science, which took place in the last twenty years, the number of performed amputations is very high, and statistical surveys show its constant, though small, increase. Currently, approximately 12-13 thousand amputations are carried out annually in Poland. Each amputation decreases the quality of life in various areas. There are problems with everyday, professional, social and mental activities.
That is why, the development of orthopedic prosthetics which aims at restoring the function of the lost limb as best as possible, is so important.

The present level of technological advancement allows manufacturing the prostheses of upper limbs, which can replace the lacking limb, to a certain degree. Their functions may be purely visual (esthetic - cosmetic prostheses), but also fully operational - controlled mechanically or electronically (so-called bionic prostheses). A prosthesis, as an artificial replacement of a missing body part, is manufactured in several steps. Their total realization time can take, depending on the particular prosthesis type, between one week and 3 months.

For an average patient, a problem in accessing these devices is their price, which is proportional to technological advancement and quality of production of a given prosthesis. The time of obtaining a prosthesis is also an important factor, especially in the case of injuries and small children, where several weeks or even months can be much too long from the point of view of both therapeutic and psychic comfort of the patient. It is also important that 
fitting a prosthesis to a given patient is not a simple task. As certain studies indicate, there is often a problem in the mutual communication between a patient and a prosthetist, which it can negatively affect the final satisfaction of using a given prosthesis [2].

As an attempt to solve or reduce the scale of the above-mentioned problems and limitations, it is proposed to use an alternative process of manufacturing, based on 3D scanning of patient limbs and use of low-cost additive manufacturing technologies, also known as the 3D printing. It allows a relatively quick obtaining of the products of complex geometry, such as anatomic shapes. The greatest benefits of additive manufacturing, widely used, for example, in automotive and machine industry, are achieved with prototype and single piece production [3]. The production of prostheses adjusted to the anthropometric features of a particular patient's body is exactly of this character. That is why, additive manufacturing is often used in medical purposes, for external products (orthoses [4], prostheses [5]) and implants [6], as well as pre-surgery and mid-surgery supplies [7].

What is more, the additive manufacturing combined with the reverse engineering approach allows less problematic (in comparison to the traditional approach) processing, storing and transferring of the anthropometric data, which makes it easier to repair prostheses by manufacturing the parts which are worn out or damaged. The whole digital approach to the process should bring the costs down considerably and improve the time of obtaining of the ready product, while eliminating the need for manual skills of the prosthetic technicians, required in the traditional process [8].

The most important part of any upper limb prosthesis is its socket. If it is not properly adjusted, the patient may experience pain, ulceration and blisters, and the prosthesis will be troublesome. For many people after the loss of the upper limb, the use of a prosthesis is necessary at work and in private life. However, a successful prosthetic fit depends largely on the quality of the prosthetic socket. Functionality, comfort and hygiene enable the user to conduct a largely independent daily life [9].

In the case of a person shortly after amputation, the stump changes so quickly in the temporary prosthesis that the fit may require a weekly update. It can be expected that it will be necessary to replace the hopper at least once before receiving the final denture. The stump drastically shrinks during the first few months of wearing the prosthesis, and stabilization can last up to two years.

The socket is the most difficult part to manufacture in any prosthesis and, at the same time, the part which needs to be replaced most often. Even the final socket should be replaced sometimes, e.g. if it is worn out or broken or simply does not fit anymore (its user gained or lost weight or the stump is swollen etc.). Gaining an ability of rapidly manufacturing sockets on the basis of contactless measurements would allow a significant increase in comfort of the patients and availability of more advanced prosthetics.

The 3D printing techniques are generally a known approach in the process of creation and testing of prosthetic sockets. Various attempts have been made in the recent years. As early as 2005, Herbert et. al. presented a results of preliminary investigation into the topic - they successfully created a socket using a powder 3D printing machine [10]. In 2011, Chimento et al. presented the results of the 3D printing use for making tooling (molds) for traditional thermoformed devices [11]. Laszczak and others used 3D printing for creation of a special sensor for testing the stump-socket interfaces [12]. A special report by Farina \& Amsuss indicates that rapid prototyping is a promising direction in the manufacturing of modern upper limb prostheses [13]. Another report by Kate et al. shows a detailed state of technology in the production of $3 \mathrm{D}$ printed upper limb prostheses, listing the most important achievements, such as the RoboHand prosthesis [14]. There have been also attempts at creating organized methodologies of prototyping the prosthetic sockets [15]. Still, the range of use of cosmetic prostheses with individualized sockets manufactured with 3D printing is limited, due to a long and costly design process as well as high aesthetic and functional requirements.

This paper presents a process of rapid design and manufacturing of a socket for a young male patient with an amputated forearm. The whole process of manufacturing prototype $3 \mathrm{D}$ printed sockets for prostheses of various functions is shown along with conclusions on how to possibly improve and automate it, thus eliminating the design costs. 


\section{MATERIALS AND METHODS}

\section{Main concepts of research}

The aim of the work presented in this article was to examine the possibility of producing prosthetic sockets using a modern process, based on the 3D scanning techniques and additive manufacturing (3D printing). The manufactured product is to be developed in the shortest time possible, with the best possible material strength and taking into account the optimization of costs. The specific goals include:

- collecting the patient data and processing,

- designing a prosthetic socket,

- selecting the socket manufacturing technology and its implementation,

- comparing the selected materials used for printing.
As part of the work, it was planned to collect the data from a 25-year-old patient with an amputated forearm using the 3D scanning method. The next step was to process the obtained triangle mesh and design the shape of the prosthetic socket on it. Next, a socket model was created from three different materials, on three different machines for additive manufacturing, using different ways of dividing and orienting the model. The prototypes were subjected to post processing. All the work performed was assessed by the patient, who was to determine whether the socket was properly fitted and comfortable to use.

\section{Design process}

The first step in manufacturing the prosthetic socket was to scan the patient's stump. The scan was performed using David SLS-3 scanner. A special measuring station was built for this purpose.

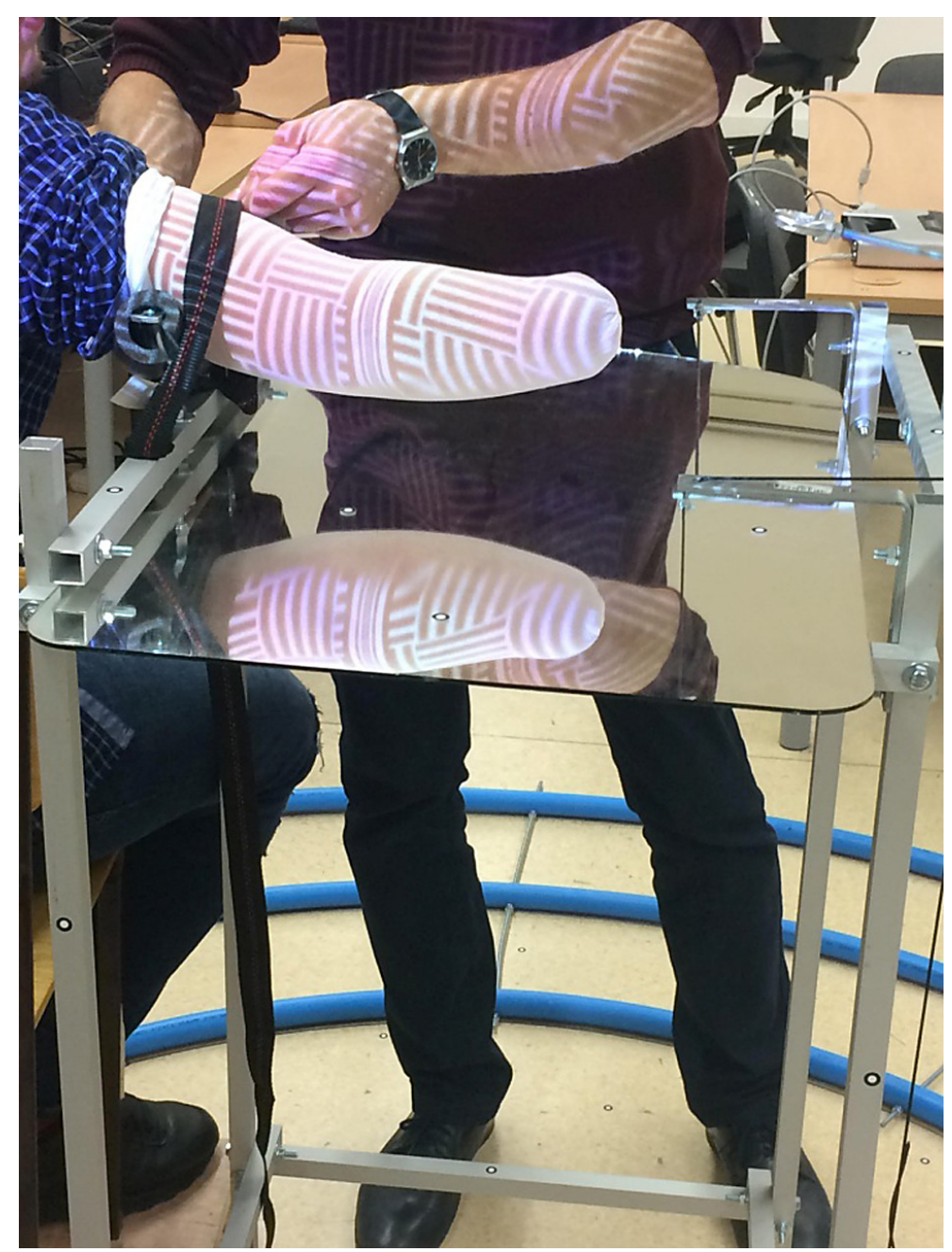

Fig. 1. Scanning of the patient's stump 
The scanner head moved through a round track, around the sitting patient with a supported fixed stump placed at an angle of $90^{\circ}$ (Fig. 1).

The first stage after 3D scanning is the assembly of partial scans and coordinate system setting. These operations were performed using the scanner software. After assembling the scan, it was necessary to cut it and repair the mesh errors in order to facilitate modeling the shape of the prosthetic socket, as well as to smoothen the surface and close holes. These changes were made in the GOM Inspect program (Figure 2).

In order to obtain a model of the prosthetic socket based on a 3D scan, the Autodesk Meshmixer program was used, to which the resulting triangle mesh representing the patient's stump was imported. The available tools were then used to create the socket shape. Separately, in Autodesk Inventor, a head of the socket was designed with a flat end, the model was imported into the Meshmixer program, and a connection with the basic geometry was made (Fig. 3).

The model was exported in STL format to Autodesk Inventor and, using the Mesh Enabler plugin, the model in STP format was obtained. Next, the mounting holes and the holes allowing the patient to put on the socket and, in the case of myoelectric hand assembly, connect it with the potentiometers placed on the patient's skin, were made. For the purpose of manufacturing in different orientations and on machines of different size of the working chamber, the socket was divided vertically as well as horizontally (Fig. 4).

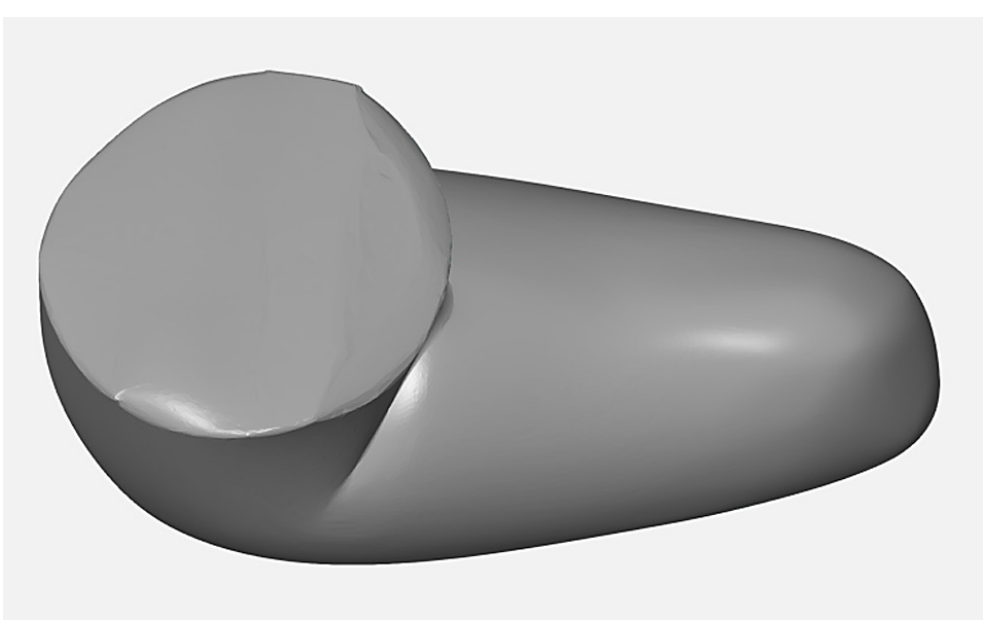

Fig. 2. Resulting triangular mesh of the patient's stump

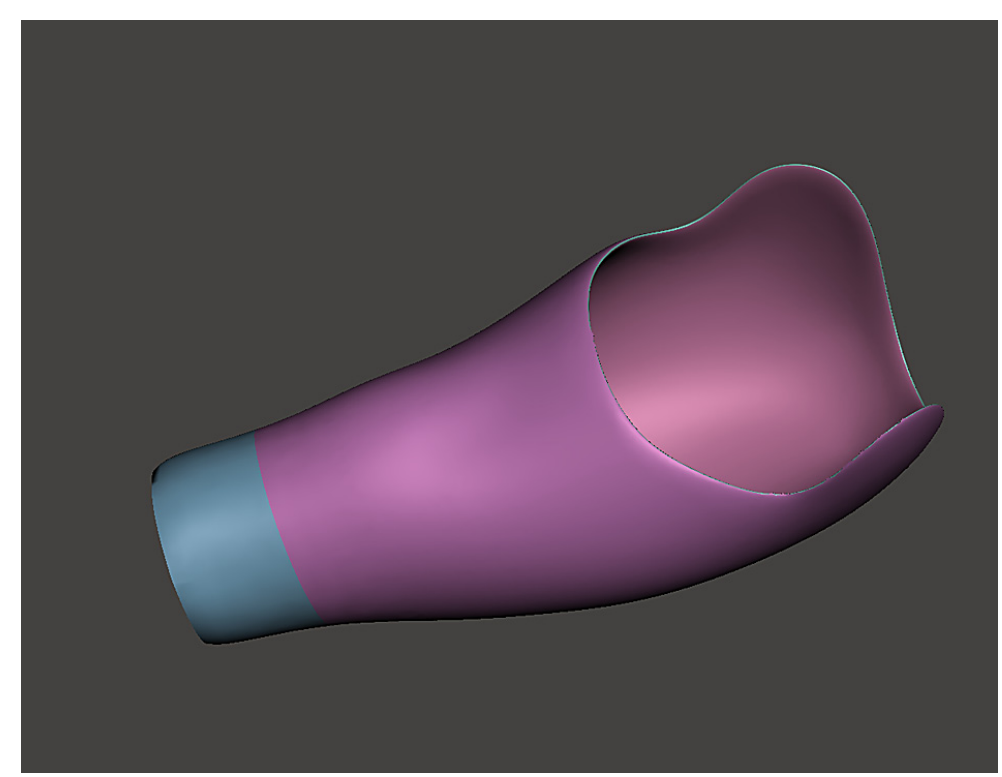

Fig. 3. Designed prosthetic socket 


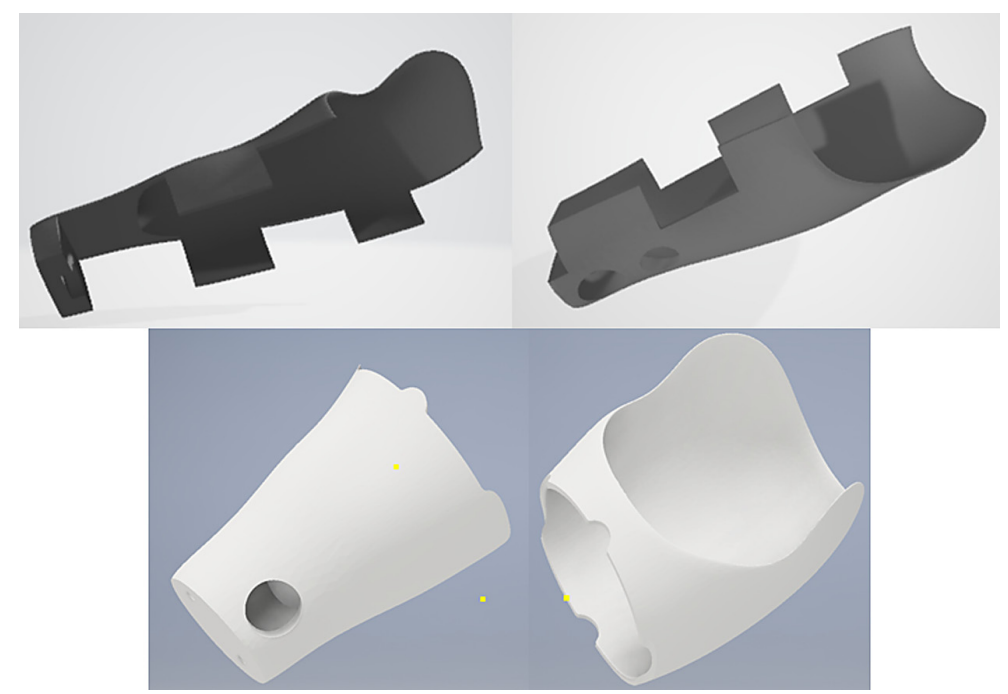

Fig. 4 Prosthetic socket in the horizontal (top) and vertical (bottom) division

\section{Additive manufacturing process}

The additive manufacturing (3D printing) was realized using the Fused Deposition Modelling process, out of thermoplastic materials. Three different materials were used: ABS, PA12 (nylon) and PC (polycarbon). The applied parameters are shown in Table 1.

As mentioned before, three different machines were used, of various price segments and capabilities. The ABS socket was manufactured as a whole (without division) using a large chamber semi-professional machine - Raise 3D Pro 2 (working chamber of $305 \times 305 \times 605 \mathrm{~mm}$ ). The PA12 socket was manufactured on a budget machine - MakerBot Replicator 2X, divided vertically. The PC socket was manufactured on a professional machine - VShaper Pro, using a horizontal division. Therefore, three different sockets were manufactured:

a) ABS, single process (no assembly), vertical orientation, Raise 3D Pro 2 machine.

b) PA12, vertical division and orientation, MakerBot Replicator 2X machine.

c) PC, horizontal division and orientation, VShaper Pro machine.
After the manufacturing, the post processing was applied. The first operation was the removal of the support structures (if present). Then, assembly was performed for the $\mathrm{B}$ and $\mathrm{C}$ models. The B model was welded by a hot knife using a nylon filament as filler. The $\mathrm{C}$ model was glued using a cyanoacrylate. The connecting surfaces, after binding, were machined to achieve a proper surface quality and improve the aesthetics.

The inside of the sockets was filled with the $2 \mathrm{~mm}$ thick EVA foam. The assembly adapters (made of ABS) were glued to the front surface and ABS plugs (manufactured separately) were placed inside the cable hole. The finished sockets were all given to a patient for testing and the opinions on them were gathered.

\section{RESULTS AND DISCUSSION}

The obtained socket parts after initial post processing are presented in Figure 5, while the patient wearing the finalized sockets is shown in Figure 6.

While comparing the obtained products, it can be concluded that it is most beneficial to

Table 1. Parameters of additive manufacturing processes for various materials used

\begin{tabular}{|c|c|c|c|c|}
\hline \multirow{2}{*}{ Material } & \multicolumn{3}{|c|}{ Parameters } & Layer thickness [mm] \\
\cline { 2 - 5 } & Extrusion temperature $\left[{ }^{\circ} \mathrm{C}\right]$ & Extrusion speed $[\mathrm{mm} / \mathrm{s}]$ & Infill [\%] & 0.3 \\
\hline ABS & 230 & 60 & 40 & 0.3 \\
\hline PA12 & 245 & 50 & 15 & 0.2 \\
\hline PC & 280 & 37.5 & 50 & \\
\hline
\end{tabular}




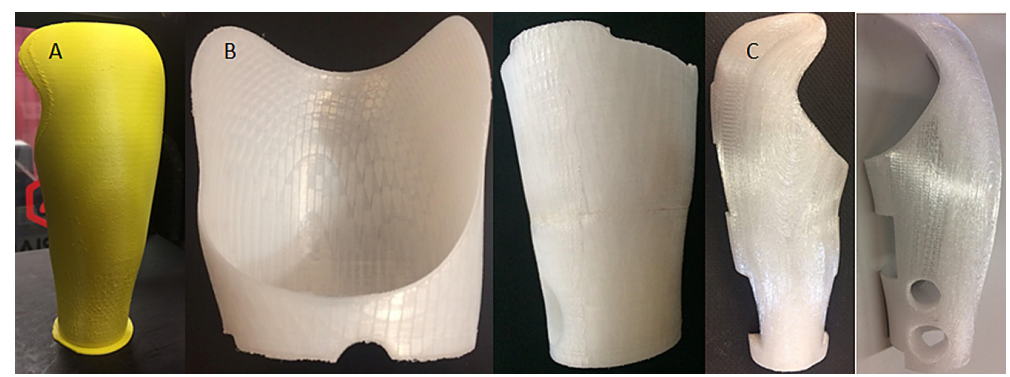

Fig. 5 Parts of manufactured sockets, A) ABS socket, B) PA12 socket, C) PC socket

manufacture the models as whole, without dividing them into parts. Attempting to connect the model halves means that the most risk of damage to the socket may occur in this place during use. An additional argument is the production time and aesthetics of the connections made. A one-piece model set vertically prints approximately an average of 10 hours and does not require the use of supports, which also prolongs the production time. After the horizontal division, the parts were created in total about 21 hours, which is twice as long and does not

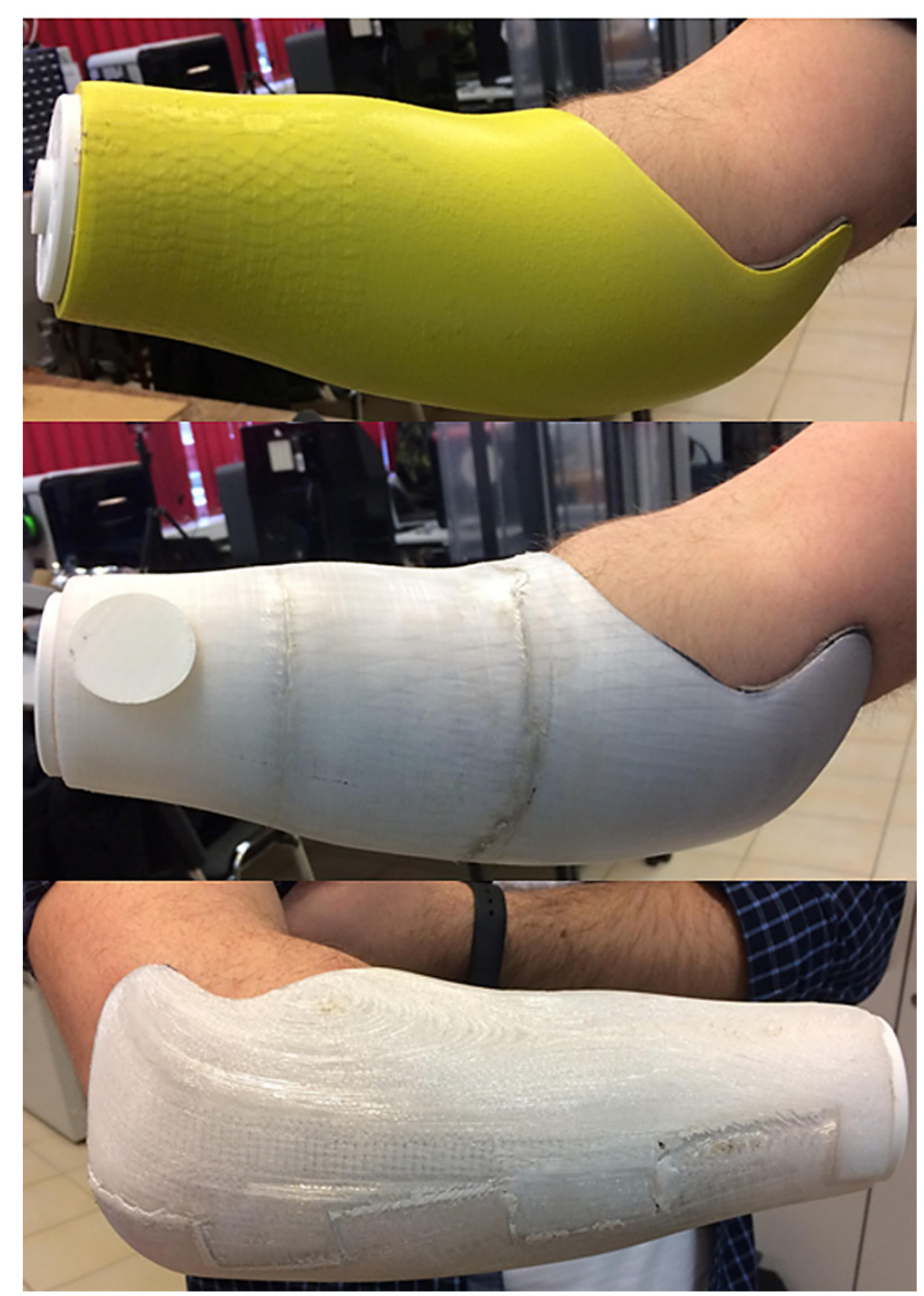

Fig. 6 Patient wearing the manufactured sockets, from top to down: A - ABS socket, B - PA12 socket, C - PC socket 
facilitate the automation of the manufacturing process (as shorter processes require less monitoring and interventions), as well as does not make the sockets more readily available for the patients. Besides, the supports had to be removed, which in the case of polycarbonate, took an additional 45 minutes for each part. In the case of vertical division, the manufacturing time was again more satisfactory, but it should be noted that this was largely influenced by the higher extrusion speed of the material during manufacture.

Comparing the materials used - the most satisfactory effect can be obtained in the case of nylon. The socket was smooth, prone to minor surface corrections and gave the impression of being the most solid when using the same wall thickness. However, this is the most demanding material in the manufacturing process. It has a tendency to unstick from the machine working table and should be firmly attached to it. Unfortunately, the effect of such a detachment can be seen on the manufactured part, but on the occasion of subsequent prints it is easy to prevent this, by properly preparing the fixing of the first layers to the table. In addition, one of the cheaper semi-professional machines existing on the market - Makerbot Replicator - has dealt with nylon. Polycarbonate, due to the necessity of using very high temperatures cannot be produced on lower class equipment, which again underlines the advantages of nylon, taking into account the costs and speed of manufacturing products of this type. The cost of a filament used to print a polycarbonate socket ( $300 \mathrm{~g}$ of material) is comparable to the cost of nylon - about 45 PLN. ABS comes out much cheaper, because $220 \mathrm{~g}$ of material was needed to make the socket, which would cost around 20 PLN.

The strength tests were not performed. However, the polycarbonate parts of the socket seemed less rigid and more prone to deformation and fracture than the nylon parts. This, however, may be a result of horizontal division, which should be abandoned in further development. The vertical division, on the other hand, can make the product more prone to fracture as a result of dynamical activity, such as hitting something hard. To sum up, there is no need of using a material with superior strength, given that the orientation and division are correctly adjusted and the process is carried out without any problems. Therefore, in terms of strength, the ABS socket manufactured as a whole is probably the best solution; however, it is not possible to achieve on most low-cost and semi-professional additive manufacturing machines.

The patient evaluated all the sockets positively; however, he had some reservations to each of them. He liked the ABS the most because of the lack of joining the individual parts, but said that the model is too loose. In addition, he drew the attention to the hard end of the socket, which arose due to the fact that the product did not use the holes needed to remove the bag used to set it up on a limb, so the machine printed the place flat. A polyamide socket was better suited to the stump; it was tighter in the elbow joint, which meant that the socket was not falling out. All the sockets were made on the basis of the same model, hence the conclusion that perhaps each of the materials had a different contraction. More looseness around the elbow may also have been caused by smoothing the edge of the socket at the modeling stage in the Meshmixer program, because during smoothing also a thinning of the wall at this point occurs. The EVA foam also turned out to be a bad idea according to the patient. Taught by the experience with previous prostheses, he stated that during the summer, foam may be unusable due to high temperatures and contact with the skin of the patient, because it is not suitable for medical applications. A kind of medical silicone would seem more pleasant to him. The model originally included the implementation of a flexible internal insert covering the stump; hence, the edges were made slightly too close to the elbow joint, which the patient also felt negatively. During the fitting, it was observed that despite using the model offset from the scan surface at the design stage, which was filled with a $2 \mathrm{~mm}$ layer of EVA foam, all the sockets had too much clearance and slipped from the patient's stump. Therefore, the problem of soft tissue compression during scanning should be focused on in the future.

Regarding the design process - Autodesk Meshmixer allows the user to quickly create the models of prosthetic sockets, using the appropriate functions. It is possible to automate the design workflow, using the available programming tools. The problem was a $90^{\circ}$ stump scan - the bend in the elbow was too large, so it would be better to manufacture it at a smaller angle, e.g. $45^{\circ}$ in the future. A large bend made it difficult to determine the socket edge during modeling. 


\section{CONCLUSIONS}

The subject of the project was the rapid manufacture of prosthetic sockets and the set goals from the patient's stump scan to the manufacturing and testing of the socket - were achieved. The activities that should be performed from the moment of scanning the anatomical part to generating the code needed to perform the manufacturing process were successfully systematized and an attempt to finish the model was made.

There have been problems with the polycarbonate socket; therefore, actions should be taken to improve the quality of products made out of this material, e.g. an attempt to change the print parameters and change the orientation of the model in the working chamber. In addition, a better method of filling the inside of the socket should be devised, so that the inner layer of the model is comfortable for the patient and suitable for the skin contact. It is possible that the solution would be to use a medical silicone or attempt to print double-material sockets (with inner layer of, e.g., TPU). This case requires many trials also involving the patient.

In the future, one should also take into account the fact that the scan performed at a smaller angle of flexion in the elbow joint will facilitate the modeling of the upper edges of the socket. In addition, the issue of compressing soft tissues should be looked into. In the case of this particular patient, there were a lot of them, which contributed to the subsequent inadequate matching of the socket. The compression stocking put on the limb was still too loose. The compression ratio that could be applied at the computer design stage of a design should be introduced.

\section{Acknowledgements}

The studies were realized with a support from Polish National Center for Research and Development, in the scope of the "LIDER" program (grant agreement no. LIDER/14/0078/L-8/16/ NCBR/2017) and statutory activity financed by Polish Ministry of Science and Higher Education (02/23/DSPB/8716).

\section{REFERENCES}

1. http://www.aotm.gov.pl/www/wp-content/uploads/taryfikacja/2016/projekty_taryf/raporty/40/ WT.521.17.2016_H72_H74_raport.pdf, access: 20.03.2017

2. Pezzin L.E., et al.: Use and satisfaction with prosthetic limb devices and related services. Archives of Physical Medicine and Rehabilitation, 2004, 85, 5, 723-729.

3. Górski F., et al.: Computation of mechanical properties of parts manufactured by fused deposition modeling using finite element method. Advances in Intelligent Systems and Computing, 2015, 368, 403-413.

4. Pallari J., et al.: Design and additive fabrication of foot and ankle-foot orthoses. Proceedings of the 21st Annual International Solid Freeform Fabrication Symposium - An Additive Manufacturing Conference, 2010, 9-11.

5. Kendall F., et al.: Development of novel 3D-printed robotic prosthetic for transradial amputees. Prosthetics and Orthotics International, 2016, 40, 3, 400-403.

6. Otawa N., Sumida T., Kitagaki H., Sasaki K., Fujibayashi S., Takemoto M., Nakamura T., Yamada T., Mori Y., Matsushita T., 2015, Custom-made titanium devices as mem-branes for bone augmentation in implant treatment: Modeling accuracy of titanium products constructed with selective laser melting, J Craniomaxillofac Surg, 43, 7, 1289-95.

7. Banaszewski J., Pabiszczak M., Pastusiak T., Buczkowska A., Kuczko W., Wichniarek R., Górski $\mathrm{F} ., 3 \mathrm{D}$ printed models in mandibular reconstruction with bony free flaps, J Mater Sci: Mater Med (2018) 29: 23

8. Cha H.Y., Lee K.H., Ryu H.J., Joo I.W., Seo A., Kim D., Kim S.J., Ankle-Foot Orthosis Made by 3D Printing Technique and Automated Design Software, Hindawi, Republic of Korea 2017

9. Sabolich S., Prosthetic Sockets, Amputee Coalition, Vol. 16, Iss. 5, September/October 2006

10. Herbert N, Simpson D., Spence W.D., Ion W., A preliminary investigation into the development of 3-D printing of prosthetic sockets, Journal of Rehabilitation Research \& Development, 2005, 42, 2, 141-146

11. Chimento J., Highsmith M.J., Crane N., 3D printed tooling for thermoforming of medical devices, Rapid Prototyping Journal, 2011, 17, 5, 387-392.

12. Laszczak P., Jiang L., Bader D.L., Moser D., Zahedi S., Development and validation of a 3D-printed interfacial stress sensor for prosthetic applications, Medical Engineering \& Physics, 2015, 37, 1, 132-137

13. Farina D. \& Amsüss S., Reflections on the present and future of upper limb prostheses, Expert Review of Medical Devices, 2016, 13, 4, 321-324

14. ten Kate J., Smit G., Breedveld P., 3D-printed upper limb prostheses: a review, Journal Disability and Rehabilitation: Assistive Technology, 2017, 12, 3, 300-314

15. Stromshed E., The Perfect Fit - Development process for the use of 3D technology in the manufacturing of custom-made prosthetic arm sockets, 2016, Lund University 